\title{
ELECTRON DAMPING IN SURFACE STUDIES
}

\author{
I. BARToš \\ Institute of Physics, Czechoslovak Academy of Sciences \\ Cukrovarnická 10, 16200 Praha 6, Czecho-Slovakia \\ (Received May 21, 1991)
}

\begin{abstract}
Electron correlation is responsible for finite lifetimes of excited electrons in crystals. Lifetime energy dependence can be obtained for infinite jellium model and only very recently the first results for an infinite crystal have been evaluated (GW approximation). Here, a phenomenological approach, based on Green functions, is presented. Broadening of local densities of electron states as well as that of angular-resolved photoemission (ARUPS) peaks and very-low-energy-electron diffraction (VLEED) profiles, due to the imaginary component of the optical potential is reviewed and interpreted. Anisotropy of electron damping on crystal surfaces has been found in VLEED as a result of electron channeling along the densely packed (111) surface atomic planes in fcc crystals. Interpretation of peak widths in VLEED-and ARUPS-profiles provides a mean to learn about damping of electrons, excited on crystal surface.
\end{abstract}

PACS numbers: 71.20.-b, 73.20. Dx

\section{Quasi-particle lifetimes}

One-particle approximation in the electron theory of crystals is surprisingly successful. This fact is explained by dressing the bare particles to form the quasi-particles with only a weak interaction (effective screening of correlation and exchange interactions).

Quasi-particles, describing excited states of the system of electrons, have been studied in detail for a simplified model of jellium, where individual positive charges of ions are replaced by a uniform positive charge background.

It has been found that the dispersion relation $E(k)$ of electrons gets somewhat modified (effective mass renormalization) and also that their lifetime (expressed as an imaginary part of electron self-energy) is in general finite. The electron lifetime is infinite at the Fermi level but shortens when electron is excited above the $E_{\mathrm{F}}$; during its collisions with other electrons, electron-hole pairs can be created, and starting from the plasmon threshold energy also collective oscillations of the system can be induced. Numerical results for such an interacting electron gas have been obtained in the random phase approximation for electron densities typical for metals [1]. 
The jellium model represents an oversimplification, of course. The density functional formalism developed later [2] represents a great step forward in the description of the electron structure of real systems. But only in the last years also a description of the excited states in the local density approximation has been successful, e.g. correct magnitudes of the band gaps of semiconductors have been obtained from the first principles in good agreement with experimental data [3]. The density functional formalism avoids the lifetimes altogether.

Only exceptionally, anothęr approximation (GW) has been successfully applied to get also the excited electron lifetimes. Therefore, it would be highly desirable to find the effective optical potential by a simpler procedure.

\section{Angular resolved photoemission peak widths}

Photoemission represents the most direct experimental evidence about crystal band structure; electrons are lifted from their occupied states to the states above the vacuum level which - after their exit from the crystal - are directly observable in vacuum. In particular, in the angular resolved photoemission mode not only their energy but also the direction of propagation is determined. In the three-step model of the photoemission process, consisting of optical (bulk) excitation, propagation to the surface and penetration of the surface barrier, the central role belongs to optical excitations among the branches of the band structure $E(k)$. Because of negligible photon momentum transitions have to be vertical (conservation of $k$ ). If then electron states are considered sharp (no interaction among electrons), the energy conservation implies that the transitions have to be possible only at discrete values of energy; the energy distributions (EDC) should then be composed of series of $\delta$-functions.

The damping is responsible for effective broadening of the $E(k)$ branches; at a small damping, the $\delta$-function in energy at a fixed $k$ is replaced by a Lorentzian. Then, the condition of energy conservation can be satisfied not only at a single wave vector $k$ but in the whole interval around, in contrast to broadening of discrete eigenvalues (e.g. in atoms), the broadening in crystals is affected by the slopes of the two bands, participating in the photoemission process. Under reasonable approximation, it can be shown that the intensity of photoemission as a function of variable parameter, (e.g. $\omega$, angle) becomes a Lorentzian of width $\bar{\gamma}$ given by $[4,5]$

$$
\bar{\gamma}=\left(\gamma_{\mathrm{h}}+\gamma_{\mathrm{e}}\left|\frac{\partial E_{\mathrm{i}} / \partial k_{\perp}}{\partial E_{\mathrm{f}} / \partial k_{\perp}}\right|\right)\left|1-\frac{\partial E_{\mathrm{i}} / \partial k_{\perp}}{\partial E_{\mathrm{f}} / \partial k_{\perp}}\right|^{-1},
$$

where $\gamma_{h}, \gamma_{e}$ represent electron and hole inverse lifetimes and the derivatives are the slopes of initial and final branches of the band structure (normal components). The peak width is thus determined by electron and hole lifetimes as well as by the slopes of the two branches (initial and final band) at a vector $k$, where energy conservation is satisfied.

If the band structure is known for both the initial occupied and final unoccupied bands in photoemission, the peak width is still determined by two inverse lifetimes $\gamma_{\mathrm{h}}$ and $\gamma_{\mathrm{e}}$. In order to determine $\gamma_{\mathrm{e}}$ from the peak width $\bar{\gamma}$, transitions 
originating just below the $E_{\mathrm{F}}$ (where $\gamma_{\mathrm{h}}$ approaches zero) are considered. The damping of unoccupied electron states has been studied by ARUPS either at fixed angle of exiting electrons, varying continuously the excitation energy (normal emission from $\mathrm{Cu}(100)$ in [4]), or at fixed energy of exciting radiation, varying the exit angle of electrons ( $\hbar \omega=16.85 \mathrm{eV}$ and $20.95 \mathrm{eV}$ from $\mathrm{Cu}(111)$ in [5]). The advantages of both approaches can be united if angle-tuning at the best photon energy is used (angle variation with synchrotron radiation in [6]). A recent collection of the lifetimes measured so far for various metals in the range to $50 \mathrm{eV}$ above $E_{\mathrm{F}}$, complemented by the results of the inverse photoemission, is given in [7]. For the interpretation of inverse photoemission data, however, the one-parameter energy dependence of the electron lifetime had to be postulated (the energy resolution is smaller than in ARUPS).

In order to determine $\gamma_{\mathrm{h}}$, the inverse lifetime of holes, the smaller effect of electron lifetimes on $\bar{\gamma}$, has been subtracted to obtain it in $\dot{u}$-bands of $\mathrm{Cu}$ [4]. Another possibility, would suggest to utilize surface states for these determinations $\left(\bar{\gamma}=\gamma_{\mathrm{h}}\right)$. These do not fit directly into the three-step formulation, however, and their treatment has to be verified. This was performed in a computer experiment [8].

With the inverse lifetimes $\gamma$, two important quantities are related:

- the lifetime $\tau$ results from the uncertainty relation $\gamma \cdot \tau=\hbar$;

- the electron mean free path $l$ is given by $l=\tau \partial E_{\mathrm{f}} / \partial k$.

The last quantity is extremely important for surface physics as it determines the thickness of the surface layer investigated by means of low energy electrons (e.g. LEED, photoelectron spectroscopy).

Electron-electron correlations represent only one, though fundamental, source of diminishing of quasi-particle lifetimes which is present even in perfect crystals.

However, any deviation from a perfect periodicity (disorder, impurities, vibrations) contributes to the broadening by relaxing the momentum conservation conditions (e.g. $\Delta k_{\|}=0$ ). Therefore, great care (experiment, samples) has to be devoted to processing of data [9]. On the other hand, it has been proposed that surface impurities induce observable broadening of peaks which can be interpreted in terms of their scattering cross-sections [10].

In a recent study, an anomalous behaviour has been observed for surface states approaching $E_{\mathrm{F}}$ at $W(001)$ [6]: the EDC width increased towards $E_{\mathrm{F}}$ instead of getting reduced. The proposed explanation introduces creation of phonons following the extraction of the electron from the interatomic band.

Also, some of the finer effects should be mentioned: a comparison of experimental data with computed peak widths as functions of $k_{\|}$[5] indicates that the simple energy dependence of lifetimes does not suffice to explain the details and their anisotropy has to be then taken into account.

The last effect can be investigated also by external electrons incident on crystal surface (LEED). 


\section{VLEED intensities}

Diffraction of low energy electrons (LEED) is used for surface crystallography; so far its use for the study of the electron structure of crystals has been rather limited. The correlations between intensities of diffracted electron beams and the relevant sections of the crystal band structure have been found in exact formulations of LEED, e.g. in the formalism of surface Green functions [11]. The singularities in the intensity profiles are mostly blurred by inelastic damping, however; only at very low energies (VLEED), the damping processes are weaker and the intensity profiles (energy dependence of the intensities) are sharper.

Correlations of the VLEED-peaks with partial band gaps of crystals have been observed for transition metals at electron energies below $30 \mathrm{eV}$ [12]. In addition, very sharp resonances have been observed there, their widths were too small to be compatible with the magnitude of electron damping there, and artificially reduced damping had to be introduced into the crystal potential in order to get a correct theoretical description of the intensities. Phenomenologically, a smaller damping for the electron beams propagating along the (111)-surface has been introduced [13] to achieve this goal.

In order to explain the sharpness of resonances in electron reflectivity from (111) surfaces of transition metals, several theoretical investigations have been undertaken, starting with a simple one-dimensional nearly-free-electron model.

The role of inhomogeneity of electron damping has been shown [14] to increase in the vicinity of the Brillouin zone boundary. It is manifested characteristically in partial electron densities of states $D(k, E)$ (density of states with fixed wave vector $k$ ) depending on relative displacement of the real and imaginary components of the crystal potential, either a singularity below the gap gets sharpened and that above the gap gets broadened (out-of-phase case) or the asymmetry is reversed. Consequences of this asymmetry for the reflectivity of the electron beam have been shown in [15]; the reflectivity maximum is displaced from the energy, corresponding to the centre of the gap upwards or downwards. Similar behaviour has been found numerically also for the specular beam reflectivity from the copper surface $\mathrm{Cu}(100)$ (for normal incidence). These peak shifts, being restricted to take place only within the band gap, are rather small, however. Bigger effects are to be expected if there are pronounced structures in the vicinity of the band gap (outside the gap). Such a case, which is typical for all fcc transition metals, has been investigated for VLEED from $\mathrm{Cu}(111)[13,16]$. There, the peak under the main Bragg-peak remains pronounced when asymmetric damping is switched on (resonance peak) whereas the peak above disappears in the background.

The reason for the decrease of absorption along the surface consists in spatial distribution of incident electrons (at energies close to the Bragg-peak) [17, 18]. It gets predominantly concentrated into planes of atomic nuclei and so the interaction with valence electrons of the crystal is effectively reduced and "channeling" of incident electrons along the surface takes place similarly as it is known from diffraction studies at high electron energies (Borrmann effect).

Low energy electron diffraction which - in contrast to photoemission process - takes place at single energy can thus complement ARUPS studies (being $N+1$ 
electron instead of $N$ electron problem) and bring also evidence about anisotropy of damping in the vicinity of crystal surfaces.

\section{References}

[1] B.I. Lundqvist, Phys. Status Solidi 32, 273 (1969).

[2] N.D. Lang, in: Solid State Physics Eds. H. Ehrenreich, F. Seitz D. Turnbull, Vol.28, Academic Press, New York 1973, p. 225

[3] M. Schlütter, L.J. Sham, in: Advances in Quantum Chemistry 21, Academic Press, 97 (1990).

[4] J.A. Knapp, F.J. Himpsel, D.E. Eastman, Phys. Rev. B 19, 4952 (1979).

[5] J.K. Grepstad, B.J. Slagsvold, I. Bartoš, J. Phys. F 12, 1679 (1982).

[6] S. Dhar, S.D. Kevan, Phys. Rev. B 41, 8516 (1990).

[7] W. Altmann, Dissertation, Max-Planck-Institut für Plasmaphysik, Garching 1988.

[8] I. Bartoš, J. Koukal, Surf. Sci. 183, 21 (1987).

[9] S. Kevan, Phys. Rev. B 28, 4822 (1983).

[10] J. Tersoff, S.D. Kevan, Phys Rev. B 28, 4267 (1983),

[11] B. Velický, I. Bartoš, in: LEED-Surface Structure of Solids, JĆSMF Praha, 423 (1972).

[12] R.C. Jaklevic, L.C. Davis, Phys. Rev. B 26, 539 (1982).

[13] J.C. Bossé, J. Lopez, Surf. Sci. 162, 953 (1985).

[14] I. Bartoš, Phys. Status Solidi B 122, K159 (1984).

[15] I. Bartoš, J. Koukal, in: Springer Series in Surface Sciences 2, 1132 (1985).

[16] I. Bartoš, J. Koukal, Surf. Sci. 183, 21 (1987).

[17] I. Bartoš, J. Koukal, Progr. Surf. Sci. 35, 55 (1990).

[18] F. Máca, private communication. 